\title{
Multiple discrete-energy ion features in the inner magnetosphere: 9 February 1998, event
}

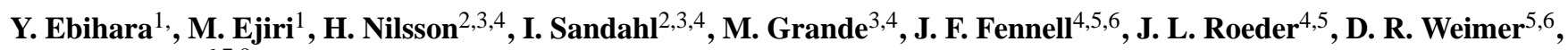 \\ and T. A. Fritz ${ }^{6,7,8}$ \\ ${ }^{1}$ National Institute of Polar Research, Tokyo, Japan \\ ${ }^{2}$ Swedish Institute of Space Physics, Kiruna, Sweden \\ ${ }^{3}$ Rutherford Appleton Laboratory, Chilton, Didcot, UK \\ ${ }^{4}$ The Aerospace Corporation, Los Angeles, California, USA \\ ${ }^{5}$ Mission Research Corp., Nashua, New Hampshire, USA \\ ${ }^{6}$ Boston University, Boston, Massachusetts, USA
}

Received: 14 May 2003 - Revised: 28 August 2003 - Accepted: 4 September 2003 - Published: 2 April 2004

\begin{abstract}
Multiple discrete-energy ion bands observed by the Polar satellite in the inner magnetosphere on 9 February 1998 were investigated by means of particle simulation with a realistic model of the convection electric field. The multiple bands appeared in the energy vs. $L$ spectrum in the $1-100 \mathrm{keV}$ range when Polar traveled in the heart of the ring current along the outbound and inbound paths. We performed particle tracing, and simulated the energy vs. $L$ spectra of proton fluxes under the dipole magnetic field, the corotation electric field, and the realistic convection electric field model with its parameters depending on the solar wind data. Simulated spectra are shown to agree well with the observed ones. A better agreement is achieved when we rotate the convection electric potential eastward by $2 \mathrm{~h}$ in MLT and we change the distribution function in time in the nearEarth magnetotail. It is concluded that the multiple bands are likely produced by two processes for this particular event, that is, changes in the convection electric field (for $>3 \mathrm{keV}$ protons) and changes in the distribution function in the nearEarth magnetotail (for $<3 \mathrm{keV}$ protons).
\end{abstract}

Key words. Magnetospheric physics (energetic particles, trapped; electric field) - Space plasma physics (numerical simulation studies)

\section{Introduction}

The Polar satellite, on many traversals of the inner magnetosphere $(L<7)$, observed multiple peaked ion bands appearing on an energy vs. time spectrum in the energy range between $\mathrm{keV}$ and hundreds keV (Peterson et al., 1998; Fennell et al., 1998). The multiple peaked ion bands are similar to the "nose" dispersion (Smith and Hoffman, 1974), which is understood to the time-dependent penetration of the plasma sheet ions coming from the near-Earth magnetotail (Ejiri et

Correspondence to: Y. Ebihara

(ebihara@nipr.ac.jp) al., 1980). The multiple bands are distinguished from the bouncing clusters of field-aligned ions (Quinn and McIlwain, 1979; Mauk, 1986; Kazama and Mukai, 2003) and the convective dispersion of field-aligned ions (Winningham et al., 1984; Bosqued et al., 1986; Frahm et al., 1986; Hirahara et al., 1996).

A classic "dip" or "gap" is often observed in the morning and dayside magnetosphere (Lyons and Williams, 1976; Lennartsson et al., 1979; Kistler et al., 1989; Shirai et al., 1997; Kovrazhkin et al., 1999; Buzulukova et al., 2002). The classic "dip" or "gap" is observed to be accompanied by a single minimum of the ion flux in a spectrum. The wedgelike dispersion, which is also observed in the inner magnetosphere in the sub-keV energy range (Yamauchi et al., 1996; Ebihara et al., 2001), may be a class of the multiple bands, but the features are somewhat different.

A sudden appearance of energetic particles is common in the inner magnetosphere. This is known to be accompanied with energy-time dispersion, and attributed to temporal changes in the ambient magnetic field configuration in the near-Earth tail (McIlwain, 1974; Mauk and McIlwain, 1974; Nagai, 1982; Lopez et al., 1990; Reeves et al., 1991; Grande et al., 1992; Li et al., 2003).

Sheldon et al. (1998) showed field-aligned beams of $40 \mathrm{keV}$ ions in the heart of the ring current $(L=3-7)$. They speculated that nose ions $(90 \mathrm{keV})$ create a parallel electric field that is responsible for the ionospheric beam features.

Modeling has been conducted to understand the formation of the multiple peaked ion bands. Peterson et al. (1998) proposed that plasma sheet ion clusters, isolated in space and time in the magnetotail, migrate sunward under the convection electric field, and form the multiple ion bands.

Fennell et al. (1998) performed a particle simulation (Chen et al., 1993) under a time-dependent convection electric field, and suggested that there are two distinct sources of the ions; the ionosphere for the lower energy bands $(\sim<1 \mathrm{keV})$ and the near-to-distant plasma sheet for the higher energy bands $(\sim>1 \mathrm{keV})$. The multiple peaks can be partially explained 
by the time-dependent transport, that is, the different energy ions take different times to reach Polor under the timedependent convection electric field. Kistler et al. (1999) and Angelopoulos et al. (2002) showed, on the basis of numerical simulation, that multiple minima of the ion flux can be produced in the sub-keV energy range when a realistic convection electric field model is employed. Multiple bands of electrons have been observed (Burke et al., 1995), and Liemohn et al. (1998) attributed them to changes in the large-scale convection electric field.

Another proposed mechanism is the substorm-associated electric and magnetic fields that inject the plasma sheet ions into the inner region rapidly. Li et al. (2000) traced ion drift trajectories by introducing an electromagnetic pulse emitted by a substorm-associated dipolarization event in the magnetotail. In their model, the pulse of electric and magnetic fields propagates from the magnetotail to $L=3.5$ at a constant velocity of $100 \mathrm{~m} / \mathrm{s}$. The pulse is partially reflected after reaching $L=3.5$. Multiple peaked spectra of ions are shown to appear without introducing the convection and corotation electric fields. They concluded that the multiple peaked spectra are fully due to a time-of-flight effect, that is, a particle with a fast drift velocity reaches a certain point first, followed by a particle with a slow drift velocity when a source population is confined spatially and temporally. This kind of propagating pulse of electric and magnetic fields associated with a substorm has been introduced to numerical tracing, to account for the sudden appearance of radiation belt electrons ( $\mathrm{Li}$ et al., 1998) and ring current ions (Li et al., 2000; Ganushkina et al., 2001).

Multiple discrete-energy ion bands can be produced by combined mechanisms that coincidently take place in the real magnetosphere. Since the results depend entirely on the assumed magnetic and electric field models, an exact solution describing the phenomena is not desired. We are interested in finding the most dominant mechanism leading to the particular multiple ion bands observed on 9 February 1998. This event has been argued by Fennell et al. (1998) and Li et al. (2000), but the argument was not fully settled regarding the essential mechanism; the convection (Fennell et al., 1998), the substorm ( $\mathrm{Li}$ et al., 2000), the migration of isolated ion clusters (Peterson et al., 1998), and the direct entry from the ionosphere (Fennell et al., 1998).

\section{Observation}

The Polar satellite was launched on 24 February 1996 into an elliptic orbit with perigee of $2 R_{E}$ and apogee of $9 R_{E}$ at $\sim 86^{\circ}$ inclination with an orbital period of $\sim 18 \mathrm{~h}$. The MICS sensor of the Charge and Mass Magnetospheric Ion Composition Experiment (Wilken et al., 1992) measures fluxes, angular distributions and charge states of the major ions $\left(\mathrm{H}^{+}, \mathrm{He}^{+}, \mathrm{He}^{++}, \mathrm{O}^{<+3}, \mathrm{O}^{>+3}\right)$ in the energy range between $\sim 1 \mathrm{keV} / \mathrm{q}$ and $220 \mathrm{keV} / \mathrm{q}$. On 9 February 1998 , the multiple discrete-energy ion bands were observed by the MICS sensor at 08:39-09:34 UT (inbound) and 11:23-13:13 UT (out- bound), as shown in the panels 1 and 2 of Fig. 1. Polar was on the morning side for the inbound path (03:4804:03 MLT) and the evening side for the outbound path (15:51-16:27 MLT). The pitch-angle averaged differential fluxes of the protons are displayed at regular intervals in $L$. We focus on the six energy bands that appear clearly in the diagrams near 45 (band 1), 28 (band 2), and $8 \mathrm{keV}$ (band 3 ) at $L=4.0$ on the inbound path, and near 32 (band 4), 18 (band 5), and $3.5 \mathrm{keV}$ (band 6) at $L=3.5$ on the outbound path. Their pitch angle distributions show that proton fluxes perpendicular to the local magnetic field dominate fluxes parallel to the magnetic field. This suggests that the protons are distinguished from ion beams emitted directly from the conjugate ionosphere (Sheldon et al., 1998). The bands that appear on the outbound path (panel 2) are described in detail by Fennell et al. (1998).

The IMF and the solar wind data from the MFI (Lepping et al., 1995) and the SWE (Ogilvie et al., 1995) instruments aboard the Wind satellite are plotted in Fig. 2. As can be seen, the multiple ion bands were observed when the IMF$B_{z}$ was nearly zero, on the average, and $D_{s t}$ ranged between 1 and $-22 \mathrm{nT}$. This means that the magnetosphere was relatively quiet. A noticeable magnetic activity was recorded half of a day prior to the measurement. The IMF $B_{z}$ turned southward at $\sim 16: 00$ UT on 8 February, and the negative IMF $B_{z}$ lasted $9 \mathrm{~h}$. A consequent decrease in $D_{s t}$ started at 17:00 UT on 8 February, and its minimum reached $-23 \mathrm{nT}$ at 23:00 UT on 8 February.

\section{Model}

Bounce-averaged drift trajectories of protons were traced in the dipole magnetic field, the corotation electric field, and the convection electric field model. The bounce-averaged drift velocity in the dipole magnetic field is described by (Ejiri, 1978)

$$
\left\langle\boldsymbol{V}_{s}\right\rangle=\frac{\boldsymbol{B}}{B^{2}} \times\left(\nabla \Phi+\nabla \Phi_{c}+\frac{K G\left(y_{0}\right)}{q B} \nabla B\right),
$$

where $B$ is the magnetic field, $\Phi$ the convection electric potential, $\Phi_{c}$ the corotation electric potential, $K$ the kinetic energy, $q$ the charge, $y_{0}$ sine of the equatorial pitch angle, and $G$ is the function given by Ejiri (1978). $G$ is 1.0 for the equatorial pitch angle of $90^{\circ}$ and 0.85 for $30^{\circ}$. Assuming that the first two adiabatic invariants are conserved, the change in the equatorial pitch angle due to a drift in the radial direction is written by

$\frac{d y_{0}}{d t}=-\frac{y_{0} I\left(y_{0}\right)}{4 \mathcal{F}\left(y_{0}\right)} \frac{1}{R} \frac{d R}{d t}$,

where $R$ is the radial distance from the center of the Earth. The functions $I\left(y_{0}\right)$ and $\mathcal{F}\left(y_{0}\right)$ are also given by Ejiri (1978).

Two different empirical models of the convection electric field were employed in the calculation. One is the model developed by Weimer (2001) (hereinafter referred to the W2K model). Another is the Volland-Stern type model (Volland, 


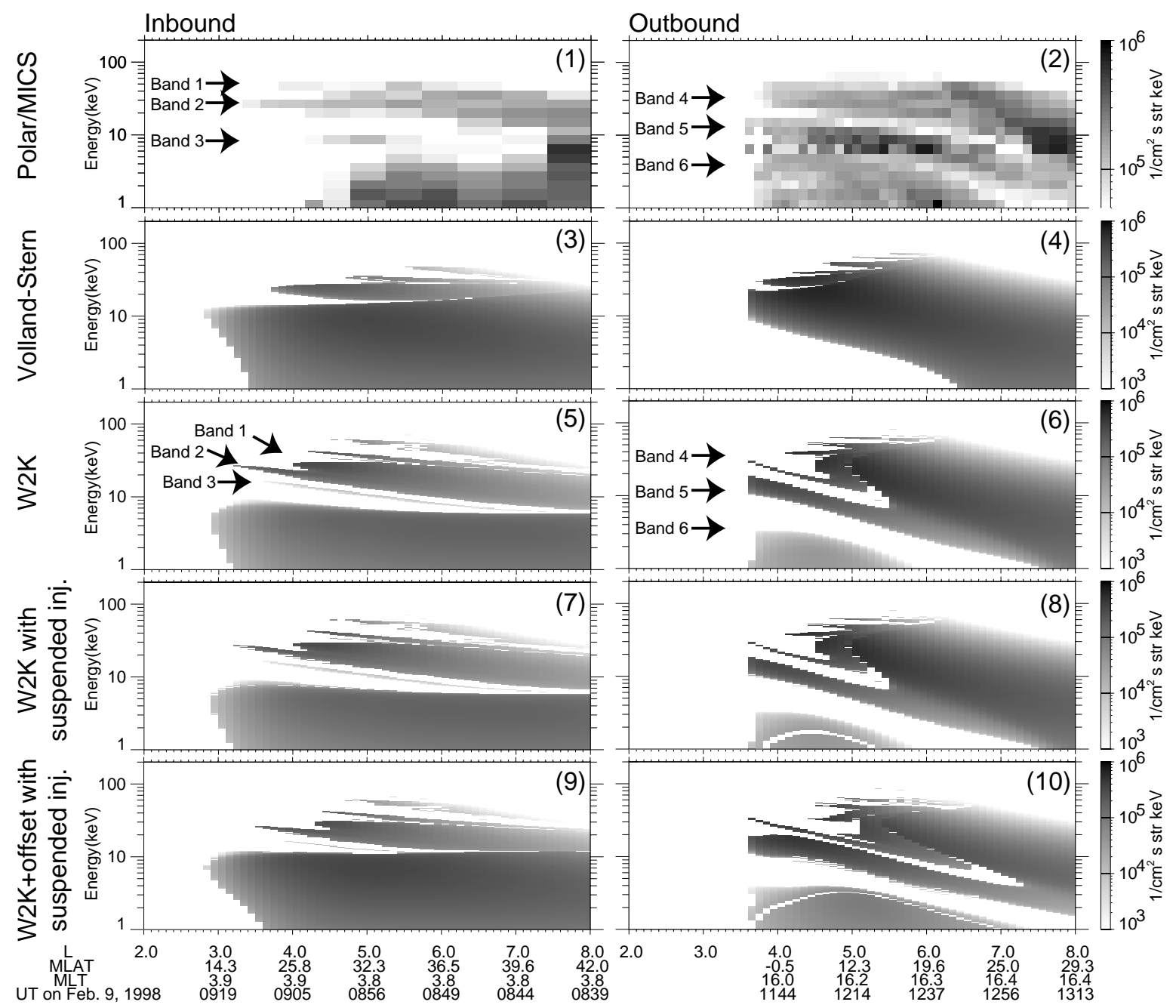

Fig. 1. From top to bottom, energy vs. $L$ spectra of pitch-angle averaged proton differential flux measured by POLAR/CAMMICE/MICS on 9 February 1998 (panels 1 and 2), energy spectra of locally mirroring proton flux calculated with the Volland-Stern type convection electric field model (panels 3 and 4), same as panels 3 and 4, except that the spectra were calculated with the W2K model (panels 5 and 6), same as panels 5 and 6, except that the spectra were calculated with suspended injection during the period between 14:00 and 16:00 UT on 8 February (panels 7 and 8), same as the panels 7 and 8, except that the W2K model is rotated eastward by 2 hours in MLT (panels 9 and 10). The left column is spectra for the inbound path and the right column for the outbound path.

1973; Stern, 1975). The W2K model gives an ionospheric electric potential depending on the solar wind velocity, IMF, and the dipole tilt angle of the Earth's rotation. The electric potential is expressed by a spherical harmonic series as

$\Phi(\phi, \theta)=\sum_{l=0}^{4} \sum_{m=0}^{\min (l, 3)}\left(A_{l m} \cos m \phi+B_{l m} \sin m \phi\right) P_{l}^{m}(\cos \theta)$

where $\phi$ is the MLT, $\theta$ is the geomagnetic colatitude, and $P_{l}^{m}$ is the associated Legendre function. The spherical harmonic coefficients $A_{l m}$ and $B_{l m}$ were derived with a least error fit, with satellite measurement done by the Dynamics Explorer-2 satellite on 2645 polar cap passes. Following the manner performed by Weimer (2001), we used a sliding average of IMF and solar wind data. The averages were taken for the 20-min period prior to the polar observation. Before averaging, the IMF and solar wind data was delayed by the travel time to the Earth. Assuming that a field line is equipotential, we mapped the ionospheric potential to the equatorial plane along the field line.

We also used the Volland-Stern type model for the purpose of reference;

$\Phi(\phi, L)=\Phi_{P C P} \frac{\sin \phi}{2}\left(\frac{L}{L_{B}}\right)^{2}$,

where $\Phi_{P C P}$ is the polar cap potential drop, and $L_{B}$ is the $L$ value at the magnetopause at dusk and dawn. $L_{B}$ is assumed to be $10.47 R_{E}$ in this particular simulation. The polar cap potential drop was calculated with the empirical model of the relationship between the solar wind parameters and the polar cap potential drop (Boyle et al., 1997) as

$\Phi_{P C P}=1.1 \times 10^{-4} V_{s w}(\mathrm{~km} / \mathrm{s})^{2}+$ 


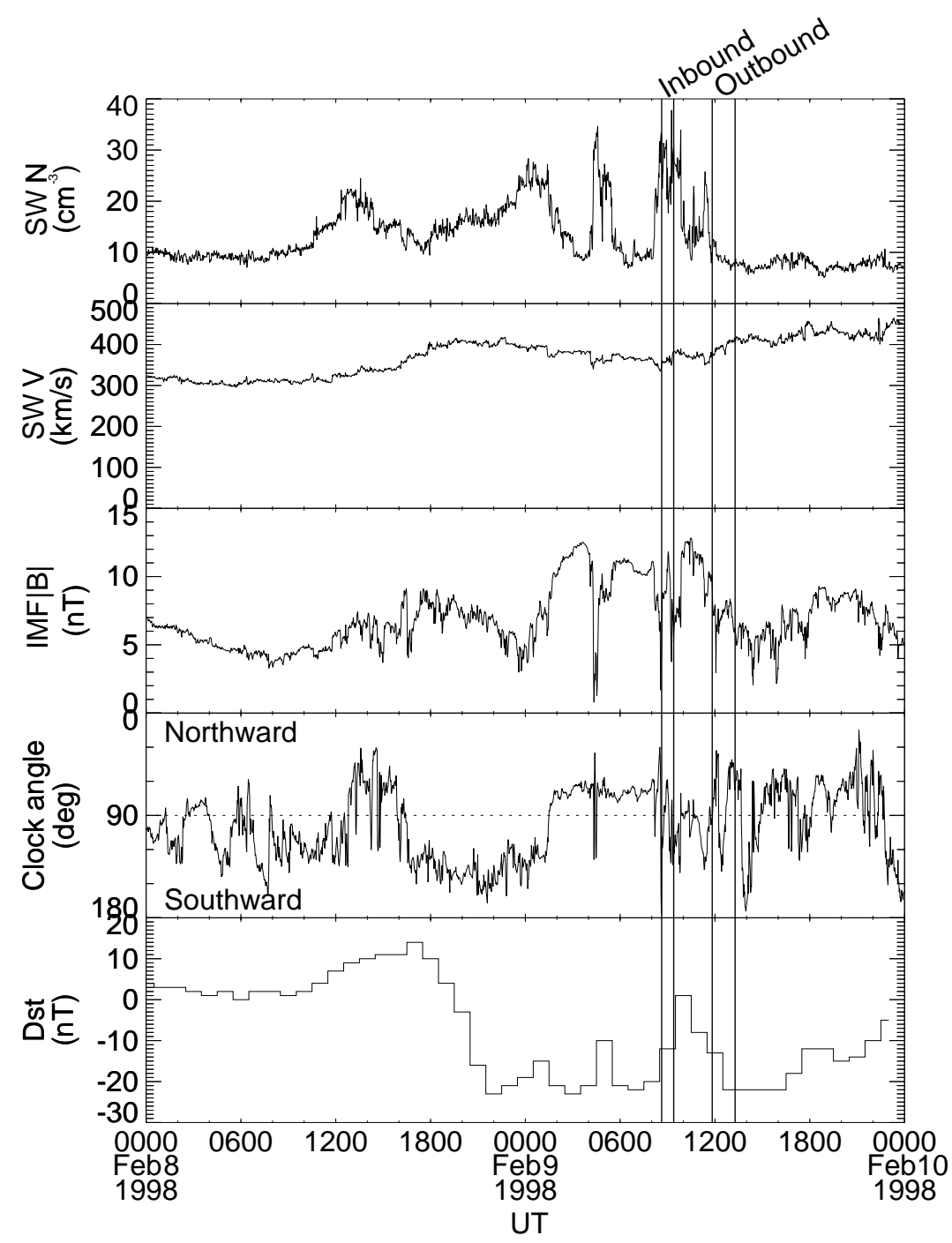

Fig. 2. From top to bottom, the solar wind density (SW N), the solar wind velocity (SW V), the intensity of the IMF (IMF B), the clock angle of IMF and $D_{s t}$ on 8-9 February 1998.

$$
11.1 B_{I M F}(\mathrm{nT}) \sin ^{3} \frac{\theta_{I M F}}{2}(\mathrm{kV}) \text {. }
$$

The differential flux along the Polar orbit was calculated with the same manner that Ebihara et al. (2001) performed. We started tracing at a satellite position specified by a fivedimensional phase space, that is, $L, \phi, K$, magnetic latitude $\lambda$, and sine of the local pitch angle $y$. Assuming that there is no parallel electric field, we can reduce the number of the dimensions from five to four, that is, $L, \phi, K$ and $y_{0}$. Particle tracing backward in time was performed until it reached the outer boundary of $10 R_{E}$, or the elapsed time exceeded $36 \mathrm{~h}$. According to Liouville's theorem, the phase space densities at the two points, the satellite position and the boundary position, are equal except for loss effects, that is,

$\left.f\left(L\right.$, MLT, $\left.K, y_{0}\right)\right|_{\text {satellite }}=\left.f\left(L\right.$, MLT, $\left.K, y_{0}\right)\right|_{\text {boundary }}-\Delta f$,

where $\Delta f$ denotes the loss of the phase space density. The charge exchange loss with the neutral hydrogen contributes to $\Delta f$ in the calculation, and is calculated along the bounceaveraged drift trajectory as

$\Delta f=\int \frac{\left.f\left(L, \operatorname{MLT}, K, y_{0}\right)\right|_{\text {boundary }}}{\left.\bar{\tau}\left(L, K, y_{0}\right)\right|_{\text {trajectory }}} d t$,

where $\bar{\tau}$ is the bounce-averaged lifetime of the charge exchange (c.f. Ebihara et al., 2001). The Hodges (1994) model with a $F_{107}$ value of 80 was employed for the neutral hydrogen density, and the cross section of the charge exchange reaction was given by Janev and Smith (1993). The local pitch angle of $90^{\circ}$ at the Polar latitude was taken into account in the calculation. This means that the equatorial pitch angle for a fixed local pitch angle varies along the orbital paths because of high inclination of the orbital plane. For the dipole magnetic field, the sine of the equatorial pitch angle $y_{0}$ is 


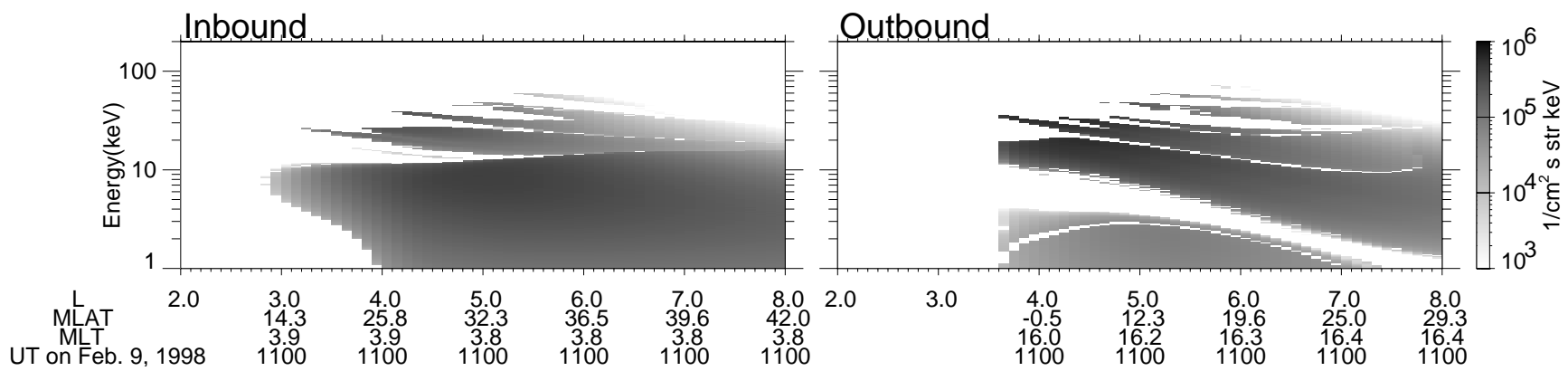

Fig. 3. Same as panels 9 and 10 of Fig. 1, except that time is fixed to 11:00 UT on 9 February 1998.

given by

$y_{0}=y \frac{\cos ^{3} \lambda}{\left(1+3 \sin ^{2} \lambda\right)^{1 / 4}}$,

where $y$ is the sine of the local pitch angle.

The phase space density at the outer boundary $(L=10)$ was assumed to be isotropic Maxwellian, with a temperature of $2 \mathrm{keV}$ and a density of $0.1 \mathrm{~cm}^{-3}$. The phase space density at the boundary $(L=10)$ does not significantly modify the result because we focus, in particular, on the formation of the banded structure, and not the absolute intensity of the fluxes.

\section{Results}

\subsection{Volland-Stern convection model}

Spectra of proton flux calculated along the Polar orbit under the Volland-Stern type convection electric field model are shown in panels 3 and 4 of Fig. 1. A traditional "nose" dispersion (Smith and Hoffman, 1974) is displayed on the inbound and outbound paths. Additionally, fine-scale structures are seen to be attached to the high energy portion of the "nose" dispersion. The fine-scale structures may be associated with the multiple bands observed by Polar/MICS, but the features are somewhat different from the observed ones, especially on the outbound path. First, band 6 is invisible in the calculated spectra. Second, the high energy edge of the "nose" structure increases with $L$, but the high energy edge of the band 5 decreases with $L$.

\subsection{W2K convection model}

Calculated spectra under the W2K model are shown in panels 5 and 6 of Fig. 1. In comparison with the spectra under the Volland-Stern model, the multiple bands, which are similar to the observed bands 1-5, are clearly presented. This suggests that the changes in the large-scale convection electric field are sufficient to produce the multiple discrete-energy ion features, at least, observed by Polar/MICS on 9 February 1998.

The low energy feature $(<3 \mathrm{keV})$ on the outbound path (panel 6 of Fig. 1) is similar to the observed band 6 (panel 2 ), but no banded feature is apparent. The disagreement is resolved when we suspend the injection at $L=10$ for a while. The particle tracing determines a time window in which the low energy protons $(<3 \mathrm{keV})$ observed on the outbound path departs at the outer boundary $(L=10)$, that is, between $\sim$ 12:00 UT and 20:00 UT on 8 February. When we suspend the particle injection, for example, between 14:00 UT and 16:00 UT on 8 February, a banded feature, which resembles the observed band 6 , appears in the spectrum as shown in the panel 8. It may be safe to say that band 6 is attributed to a change in the distribution function (e.g. decrease in the number density) in the near-Earth magnetotail. Of course, we cannot determine the absolute time of the period of the suspended injection, because it depends entirely on the convection electric field and magnetic field models assumed. Band 6 may be a class of the type 2 wedge-like dispersion which is often seen in the dayside magnetosphere (Ebihara et al., 2001).

Panel 6 of Fig. 1 exhibits that bands 4 and 5 seem to be merged at $L=5.1$, while the merging occurs at $L=7.3$ for the observation (panel 2). We achieve a better agreement analogically between them when we rotate the W2K convection potential slightly eastward as shown in the panel 10 . The merging occurs at $L=7.3$ when we rotate the $\mathrm{W} 2 \mathrm{~K}$ convection potential eastward by $2 \mathrm{~h}$ in MLT.

Figure 3 also shows the calculated spectra, but the time was fixed at 11:00 UT on 9 February 1998. The W2K convection potential was rotated by $2 \mathrm{~h}$ eastward in MLT, and the injection was suspended between 14:00 and 16:00 UT on 8 February 1998. The presence of the multiple bands means that the multiple-bands are primarily due to spatial effect, rather than temporal effect (i.e. the time-of-flight effect, as suggested by Li et al., 2000).

\section{Discussion}

On the close analogy between the observed and calculated spectra, the multiple bands observed on 9 February 1998 are probably understood as follows; the change in the large-scale convection electric field driven by the solar wind and IMF for bands $1-5$, and the change in the distribution function of the plasma sheet for band 6 . The particle tracing indicates that bands $1-5$ consist of protons coming from the near-Earth magnetotail passing through the outer boundary $(L=10)$ by 


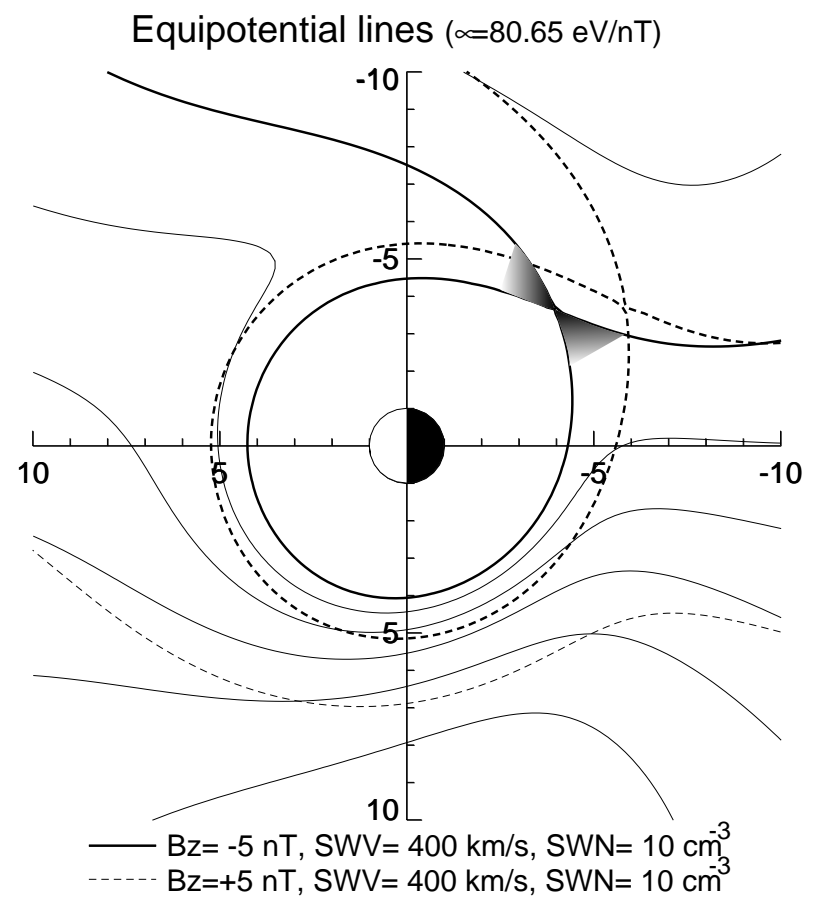

Fig. 4. Possible explanation for the ion gaps observed by Polar/MICS on 9 February 1998. Equipotential lines of the equatorially mirroring ions with the magnetic moment $\mu$ of $80.65 \mathrm{eV} / \mathrm{nT}$ (corresponding to the kinetic energy of $20 \mathrm{keV}$ at $L=5$ ) under the corotation electric potential and the $\mathrm{W} 2 \mathrm{~K}$ convection potential for the southward IMF (solid line; IMF $B_{z}$ of $-5 \mathrm{nT}$, the solar wind velocity of $400 \mathrm{~km} / \mathrm{s}$ and the solar wind density of $10 \mathrm{~cm}^{-3}$ ) and the northward IMF (dashed line; IMF $B_{z}$ of $+5 \mathrm{nT}$, the solar wind velocity of $400 \mathrm{~km} / \mathrm{s}$ and the solar wind density of $10 \mathrm{~cm}^{-3}$ ) are plotted at the interval of $10 \mathrm{kV}$. Separatrix between the open and closed drift paths is indicated by a thick line. IMF $B_{x}$ and $B_{y}$ were kept constant at zero.

way of dusk, while band 6 consists of protons coming from the tail by way of dawn. The transition between band 5 and 6 corresponds to the classic "dip" or "gap" (Jordanova et al., 1999, 2001a, b).

An interpretation is needed for the physical understanding of the mechanism leading to the multiple discrete-energy bands as follows. Figure 4 shows an example of equipotential lines, satisfying $q \Phi+\mu B=$ const, where $\mu$ is the magnetic moment, for the magnetic moment of $80.65 \mathrm{eV} / \mathrm{nT}$ under the modeled electric potential for the southward IMF (IMF $B_{z}=-5 \mathrm{nT}$; solid line) and the northward IMF (IMF $B_{z}=+5 \mathrm{nT}$; dashed line). These lines are equal to the drift trajectory of equatorially mirroring ions with the same magnetic moment of $80.65 \mathrm{eV} / \mathrm{nT}(20 \mathrm{keV}$ at $L=5)$. During the period of the southward IMF (solid line), the open-closed boundary is at $L=4$ at 18:00 MLT, and the plasma sheet ions drift inward along the equipotential lines and approach the open-closed boundary. It has to be noted that it takes infinite time to reach the open-closed boundary from the near-Earth tail, especially near the stagnation point, where the sum of the drift velocities is near zero. Consequently, a "gap", which is schematically drawn by the shaded region in Fig. 4, appears near the stagnation point.

When IMF $B_{z}$ turns northward, the ions start to follow the equipotential lines indicated by the dashed lines. The openclosed boundary is extended, and the "gap" drifts westward by the grad-B and the curvature drifts. The satellite placed on the closed trajectory would detect the "gap" periodically.

We can generalize the relationship among the energy of the "gap", $L$, and the traveling time of an ion. The bounceaveraged drift velocity of the curvature and grad-B drifts under the dipole magnetic field is written by (Ejiri, 1978)

$\frac{d \phi}{d t}=-\frac{3 G\left(y_{0}\right) L K}{q k_{0} a^{2}}, \quad$ (positive eastward),

where $k_{0}$ and $a$ are the magnetic field at $L=1$, and the Earth's radius, respectively. The MLT $\phi$ at an elapsed time $T$ is

$\phi=\phi_{0}+\frac{d \phi}{d t} T$,

where $\phi_{0}$ is the MLT at $T=0$, corresponding to the moment when the convection electric field is weakened. Substituting Eq. (7) into Eq. (8) and assuming that the stagnation point takes place at 06:00 MLT $(\phi=\pi / 2-2 \pi j$, where $j=1,2,3 \ldots)$, we obtain

$K_{j}=\frac{q k_{0} a^{2}}{3 G\left(\alpha_{0}\right) L T}\left(\phi_{0}-\frac{\pi}{2}+2 \pi j\right)$, where $j=1,2,3 \ldots$

The kinetic energy $K_{j}(j=1,2,3 \ldots)$ gives the discrete energy of the "gap". The interval of the gap is

$$
\begin{aligned}
\Delta K & =\frac{2 \pi q k_{0} a^{2}}{3 G\left(\alpha_{0}\right) L T}(\mathrm{MKS}) \\
& =\frac{732}{G\left(\alpha_{0}\right) L T \text { (hour) }}(\mathrm{keV}) \text { for singly charged ion. }
\end{aligned}
$$

For example, substituting $L$ of 5, $T$ of $9 \mathrm{~h}$ and $\alpha_{0}$ of $90^{\circ}$ into Eq. (10), we obtain $\Delta K=16 \mathrm{keV}$ for singly charged ions. The Eqs. (9) and (10) expect that the energy of the "gap" decreases with increasing $L$, and that the energy interval $\Delta K$ decreases with increasing the elapsed time $T$ (or $L$ ). The equations also expect that the feature of the "gap" depends on the energy/charge ratio. Unfortunately, the MICS instrument was unable to detect clearly the multiple bands of other ion species for this event.

This estimation is valid for the dipole magnetic field, but probably describes a general feature of the multiple discreteenergy bands that appear in the energy vs. $L$ spectra obtained by a satellite in the inner magnetosphere. This may be a good explanation for bands $1-4$. In addition to this, a complicated representation of the equipotential lines of the W2K model makes band 5 complicated.

Changes in the distribution function of the plasma sheet ions (e.g. decrease in number density) are expected to account for band 6 . The concept is similar to that previously proposed by Peterson et al. (1998) and Ebihara et al. (2001), that is, the migration of spatially varying plasma 
sheet ions produces the banded ions in the inner magnetosphere. Our calculation indicates that the migration is possible to produce band 6, but not entirely to produce the remain of the bands. As suggested by Peterson et al. (1998) and Ebihara et al. (2001), we can monitor remotely the history of the plasma sheet ions by investigating some of the dispersed ion structures. Indeed, a 2-h decrease in the plasma sheet density (or corresponding change in the distribution function) was predicted by our simulation scheme.

Acknowledgements. We are grateful to B. Kellett for arrangement and technical support for the use of the POLAR/CAMMICE/MICS database. The solar wind and IMF data from the Wind satellite were obtained through CDAWeb, and we are grateful to K. W. Ogilvie and R. P. Lepping for providing the data. The $D_{s t}$ index was provided from the World Data Center for Geomagnetism, Kyoto.

Topical Editor T. Pulkkinen thanks two referees for their help in evaluating this paper.

\section{References}

Angelopoulos, V., Temerin, M., Roth, I., Mozer, F. S., Weimer, D., and Hairston, M. R.: Testing global storm-time electric field models using particle spectra on multiple spacecraft, J. Geophys. Res., 107, 10.1029/2001JA900174, 2002.

Bosqued, J. M., Sauvaud, J. A., Delcourt, D.: Precipitation of suprathermal ionospheric ions accelerated in the conjugate hemisphere, J. Geophys. Res., 91, 7006, 1986.

Boyle, C. B., Reiff, P. H., and Hairston, M. R.: Empirical polar cap potentials, J. Geophys. Res., 102, 111, 1997.

Burke, W. J., Rubin, A. G., Hardy, D. A., and Holeman, E. G.: Banded electron structures in the plasmasphere, J. Geophys. Res., 100, 7759, 1995.

Buzulukova, N. Y., Galperin, Y. I., Kovrazhkin, R. A., Glazunov, A. L., Vladimirova, G. A., Stenuit, H., Sauvaud, J. A., and Delcourt, D. C.: Two types of ion spectral gaps in the quiet inner magnetosphere: Interball-2 observations and modeling, Ann. Geophys., 20, 349, 2002.

Chen, M. W., Schulz, M., Lyons, L. R., and Gorney, D. J.: Storm time transport of ring current and radiation belt ions, J. Geophys. Res., 98, 3835, 1993.

Ebihara, Y., Yamauchi, M., Nilsson, H., Lundin, R., and Ejiri, M.:Wedge-like dispersion of sub-keV ions: Particle simulation and Viking observation, J. Geophys. Res., 106, 29 571, 2001.

Ejiri, M.: Trajectory traces of charged particles in the magnetosphere, J. Geophys. Res., 83, 4798, 1978.

Ejiri, M., Hoffman, R. A., and Smith, P. H.: Energetic particle penetration into the inner magnetosphere, J. Geophys. Res., 85, 653, 1980.

Fennell, J. F., Chen, M. W., Roeder, J. L., Peterson, W. K., Trattner, K. J., Friedel, R., Livi, S., Grande, M., Perry, C., Fritz, T. A., and Sheldon, R.: Multiple discrete-energy ion features in the inner magnetosphere: Polar observations, in: Physics of Space Plasmas, MIT Center for Theoretical Geo/Cosmo Plasma Physics, Cambridge, MA, 15 395, 1998.

Frahm, R. A., Reiff, P. H., Winningham, J. D. and Burch, J. L.: Banded ion morphology: Main and recovery storm phases, in: Ion Acceleration in the Magnetosphere and Ionosphere, Geophys. Monogr. Ser., vol. 38, edited by Chang, T. S., 98-107, AGU, Washington, D. C., 1986.
Ganushkina, N. Yu, Pulkkinen, T., Bashkirov, V. F., Baker, D. N., Li, X.: Formation of intense nose structures, Geophys. Res. Lett., 28 491, 2001.

Grande, M., Perry, C. G., Hall, D. S., Wilken, B., Livi, S., Soraas, F., and Fennell, J. F.: Composition signatures of substorm injections, Proc. of ICS-1, Kiruna, Sweden, 485, 1992.

Hirahara, M., Mukai, T., Nagai, T., Kaya, N., Hayakawa, H., and Fukunishi, H.: Two types of ion energy dispersions observed in the nightside auroral regions during geomagnetically disturbed periods, J. Geophys. Res., 101, 7749, 1996.

Hodges Jr., R. R., : Monte Carlo simulation of the terrestrial hydrogen exosphere, J. Geophys. Res., 99, 23 229, 1994.

Janev, R. K., and Smith, J. J.: Cross sections for collision processes of hydrogen atoms with electrons, protons, and multiply-charged ions in Atomic and Plasma-Material Interaction Data for Fusion, Int. At. Energ. Agency, 4, 78, 1993.

Jordanova, V. K., Farrugia, C. J., Quinn, J. M., Torbert, R. B. Borovsky, J. E., Sheldon, R. B., and Peterson, W. K.: Simulation of off-equatorial ring current ion spectra measured by Polar for moderate storm at solar minium, J. Geophys. Res., 104, 429, 1999.

Jordanova, V. K., Farrugia, C. J., Fennell, J. F., Scudder, J. D.: Ground disturbances of the ring current, magnetopause, and tail currents on the day the solar wind almost disappeared, J. Geophys. Res., 106, 25, 529, 2001a.

Jordanova, V. K., Kistler, L. M., Farrugia, C. J., and Torbert, R. B.: Effects of inner magnetospheric convection on ring current dynamics: March 10-12, 1998, J. Geophys. Res., 106, 29, 705, 2001b.

Kazama, Y. and Mukai, T.: Multiple energy-dispersed ion signatures in the near-Earth magnetotail: Geotail observation: Geophys. Res. Lett., 30(7), 1384, doi:10.1029/2002GL016637, 2003.

Kistler, L. M., Ipavich, F. M., Hamilton, D. C., Gloeckler, G., Wilken, B., Kremser, G., and Stüdemann, W.: Energy spectra of the major ion species in the ring current during geomagnetic storms, J. Geophys. Res., 94, 3579, 1989.

Kistler, L. M., Klecker, B., Jordanova, V. K., Möbius, E., Popecki, M. A., Patel, D., Sauvaud, J. -A., Réme, H., Di Lellis, A. M., Korth, A., McCarthy, M., Cerulli, R., Bavassano Cattaneo, M. B., Eliasson, L., Carlson, C. W., Parks, G. K., Paschmann, G., Baumjohann, W., and Haerendel, G.: Testing electric field models using ring current ion energy spectra from the Equator-S ion composition (ESIC) instrument, Ann. Geophys., 17, 1611, 1999.

Kovrazhkin, R. A., Sauvaud, J. -A., and Delcourt, D. C.: InterballAuroral observations of $0.1-12 \mathrm{keV}$ ion gaps in the diffuse auroral zone, Ann. Geophys., 17, 734, 1999.

Lennartsson, W., Shelley, E. G., Sharp, R. D., Johnson, R. G.: Some initial Isee-1 results on the ring current composition and dynamics during the magnetic storm of 11 December 1977, Geophys. Res. Lett., 6, 483, 1979.

Lepping, R. P., Acuña, M., Burlaga, L., Farrell, W., Slavin, J., Schatten, K., Mariani, F., Ness, N., Neubauer, F., Whang, Y. C., Byrnes, J., Kennon, R., Panetta, P., Scheifele, J. and Worley, E.: The Wind Magnetic Field Investigation, Space Sci. Rev., 71, 207, 1995.

Li., X, Baker, D. N., Temerin, M., Reeves, G. D., Belian, R. D.: Simulation of dispersionless injections and drift echoes of energetic electrons associated with substorms, Geophys. Res. Lett., 25, 3763, 1998

Li, X., Baker, D. N., Temerin, M., Peterson, W. K., and Fennell, J. F.: Multiple discrete-energy ion features in the inner magne- 
tosphere: Observations and Simulations, J. Geophys. Res., 27, 1447, 2000.

Li, X., Sarris, T. E., Baker, D. N., Peterson, W. K., and Singer, H. J.: Simulation of energetic particle injections associated with a substorm on August 27, 2001, Geophys. Res. Lett., 30(1), 1004, doi:10.1029/2002GL015967, 2003.

Liemohn, M. W., Khazanov, G. V., and Kozyra, J. U.: Banded electron structure formation in the inner magnetosphere, Geophys. Res. Lett., 25, 877, 1998.

Lopez, R. E., Sibeck, D. G., McEntire, R. W., and Krimigis, S. M.: The energetic ion substorm injection boudnary, J. Geophys. Res., 95, 109, 1990.

Lyons, L. R. and Williams, D. J.: Storm-associated variations of equatorially mirroring ring current protons, $1-800 \mathrm{keV}$, at constant first adabatic invariant, J. Geophys. Res., 81, 216, 1976.

Mauk, B. H.: Quantitative modeling of the "convection surge" mechanism of ion acceleration, J. Geophys. Res., 91, 13, 423, 1986.

Mauk, B. H. and McIlwain, C. E.: Correlation of $K_{p}$ with the substorm-injected plasma boundary, J. Geophys. Res., 79, 3193, 1974.

McIlwain, C. E.: Substorm injection boundaries, Magnetospheric Physics, edited by McCormac, B. M., D. Reidel Publishing Company, Dordrecht-Holland, 143, 1974.

Nagai, T.: Local time dependence of electron flux changes during substorms derived from multi-satellite observations at synchronous orbit, J. Geophys. Res., 87, 3456, 1982.

Ogilvie, K. W., Chorney, D. J., Fitzenreiter, R. J., Hunsaker, F., Keller, J., Lobell, J., Miller, G., Scudder, J. D., Sittler Jr., E. C., Torbert, R. B., Bodet, D., Needell, G., Lazarus, A. J., Steinberg, J. T., Tappan, J. H., Mavretic, A., and Gergin, E.: SWE, a comprehensive plasma instrument for the Wind spacecraft, Space Sci. Rev., 71, 55, 1995.

Peterson, W. K., Trattner, K. J., Lennartsson, O. W., Collin, H. L., Baker, D. N., Pulkkinen, T. I., Toivanen, P. K., Fritz, T. A., Fennell, J. F., and Roeder, J. L.: Imaging the plasma sheet with energetic ions from the Polar satellite, Proc. of ICS-4, Terra Sci. Publishing, Tokyo, 813, 1998.
Quinn, J. M., and Mcllwain, C. E.: Bouncing ion clusters in the Earth's magnetosphere, J. Geophys. Res., 84, 7365, 1979.

Reeves, G. D., Belian, R. D., and Fritz, T. A.: Numerical tracing of energetic particle drifts in a model magnetosphere, J. Geophys. Res., 96, 13, 997, 1991.

Sheldon, R. B., Spence, H. E., and Fennell, J. F.: Observation of $40 \mathrm{keV}$ field-aligned ion beams, Geophys. Res. Lett., 25, 1617, 1998.

Shirai, H., Maezawa, K., Fujimoto, M., Mukai, T., Saito, Y., and Kaya, N.: Monoenergetic ion drop-off in the inner magnetosphere, J. Geophys. Res., 102, 19, 873, 1997.

Smith, P. H. and Hoffman, R. A.: Direct observation in the dusk hours of the characteristics of the storm time ring current particles during the beginning of magnetic storms, J. Geophys. Res., 79, 966, 1974.

Stern, D. P.: The motion of a proton in the equatorial magnetosphere, J. Geophys. Res., 80, 595, 1975.

Volland, H.: A semiempirical model of large-scale magnetospheric electric fields, J. Geophys. Res., 78, 171, 1973.

Weimer, D. R.: An improved model of ionospheric electric potentials including substorm perturbations and application to the Geospace Environment Modeling November 24, 1996, event, J. Geophys. Res., 106, 407, 2001.

Wilken, B., Weiss, W., Hall, D., et al.: Magnetospheric ion composition spectrometer onboard the CRRES spacecraft, J. Spacecraft and Rockets, 29, 585, 1992.

Winningham, J. D., Burch, J. L., and Frahm, R. A.: Bands of ions and angular V's: A conjugate manifestation of ionospheric ion acceleration, J. Geophys. Res., 89, 1749, 1984.

Yamauchi, M., Lundin, R., Eliasson, L., and Norberg, O.: Mesoscale structures of radiation belt/ring current detected by lowenergy ions, Adv. Space Res., 17 (2), 171, 1996. 